\title{
High-Resolution and High-Throughput Plasmonic Photopatterning of Complex Molecular Orientations in Liquid Crystals
}

\author{
Yubing Guo, Miao Jiang, Chenhui Peng, Kai Sun, Oleg Yaroshchuk, Oleg Lavrentovich, \\ and Qi-Huo Wei*
}

Well-designed molecular orientation (also called director) is at the heart of functional liquid crystal (LC) devices such as tunable Pancharatnam lens, gratings, and q-plates. ${ }^{[1-3]}$ With predesigned director fields, the stimulus-responsive deformations of LC elastomers can be preprogrammed, ${ }^{[4-8]}$ making possible various origami-inspired metamaterials and devices. ${ }^{[7,8]}$ Molecular director fields can also be designed to control colloidal assembly ${ }^{[9-11]}$ and nonlinear electrokinetic flows in LCs. ${ }^{[12-14]}$ While surface alignment of LC molecules has been a topic of extensive studies, ${ }^{[15-17]}$ only two types of techniques allow for designable director fields: One relies on nanogroves created by rubbing polymer films with atomic force microscope tips: ${ }^{[18]}$ the other is based on photoalignment, either through pixelby-pixel direct laser writing ${ }^{[5]}$ or digital micromirror photopatterning. ${ }^{[19,20]}$ These maskless techniques are ideal for fast prototyping, however their scalability for large-scale micro and nanodevices manufacturing is limited because they rely on serial patterning processes.

Photoalignment of LCs is realized with azo-dyes, polymers susceptible to photodestruction, and photocrosslinkable substances. ${ }^{[16]}$ Azo-dyes show the advantages of excellent LCs alignment with small exposure dose and rewritable alignment orientation. The essence of photoalignment relies on orientational ordering of azo-dye molecules induced by illumination of linearly polarized light, usually as a result of trans-cis photoisomerization. ${ }^{[16,17]}$ Although the underlying mechanisms may vary with the kinetics of photochemical reaction, azo molecules preferably align in the direction in which their optical absorption minimizes, ${ }^{[16,17]}$ leading to an average molecular long

Y. Guo, M. Jiang, C. Peng, Prof. O. Lavrentovich, Prof. Q.-H. Wei

Liquid Crystal Institute

Kent State University

Kent, OH 44242, USA

E-mail: qwei@kent.edu

Dr. K. Sun

Department of Material Science and Engineering

University of Michigan

Ann Harbor, MI 48109, USA

Prof. O. Yaroshchuk

Institute of Physics

National Academy of Sciences of Ukraine

Kyiv 03028, Ukraine

DOI: 10.1002/adma.201506002 axis perpendicular to the optical polarization. When LC materials are in contact with these exposed photoalignment layers, the photoinduced orientation ordering in the azo-molecules is transferred into the bulk of LCs due to the long range nature of orientational ordering.

Optical metasurfaces made of 2D nanoantenna arrays are emerging with extraordinary abilities in controlling the flow of light at subwavelength length scales. ${ }^{[21,22]}$ It has been shown that by engineering spatial variations in the shape, size, and orientation of constituent nanoantennas, all parameters of light such as phase, polarization, momentum, and intensity can be manipulated spatially with high precision, enabling numerous new functionalities and applications, including generalized Snell's law, ${ }^{[23,24]}$ vortex beam generation, ${ }^{[25-27]}$ Pancharatnam lens, ${ }^{[28]}$ holography, ${ }^{[29]}$ and aberration free ultrathin flat lenses. ${ }^{[30]}$ The capabilities of plasmonic materials in focusing light at subwavelength scales have been shown to be advantageous in nanoscale lithography where patterns of light intensities are exploited for photoreaction. ${ }^{[31,32]}$ In contrast, the exceptional capabilities of plasmonic materials in manipulating optical polarizations have never been utilized in photopatterning.

Here, we present a plasmonic photoalignment technique that enables patterning LC molecular director fields with unprecedentedly high resolution and throughputs. This plasmonic photopatterning of molecular orientation is based on engineered plasmonic metamasks (PMMs) which are made of rectangular nanoaperture arrays in Al films. When illuminated by a broadband and nonpolarized light, the PMMs generate patterns of both light intensity and polarization. By projecting the optical patterns of the PMMs onto photoalignment materials coated inside liquid crystal cells, alignment patterns encoded in the spatial orientations of the nanoapertures can be induced in the photoalignment layers and then imposed in liquid crystals. We demonstrate that LC director patterns with arbitrary complexity such as 2D and 3D topological defects can be patterned with a diffraction-limited spatial resolution and single exposure. This technique makes alignments of LC molecular orientations a repeatable and scalable process similar to conventional photolithography, which can not only pave a route to large scale manufacturing of various LC devices requiring nonuniform molecular orientations but also represents a unique application of plasmonic metasurfaces.

Ideally, the PMMs would be similar to traditional photomasks: Function in the transmission mode with dark states 
for unexposed regions, a simple design rule, a broadband optical transmission, and a high polarization contrast in the absorption band of the photoalignment materials. The two commercial photoalignment materials (brilliant yellow from Sigma and PAAD-72 from BeamCo) used in this work have absorption bands in the wavelength range between 400 and $550 \mathrm{~nm}$ (Figure S1, Supporting Information). These design requirements make it hard to employ those high transmission metasurfaces based on dielectric resonators or plasmonic antennas, ${ }^{[21,22,26,27]}$ therefore, we instead designed the PMMs by taking the advantages of the extraordinary optical transmission through rectangular nanoaperture arrays in $\mathrm{Al}$ films. Here, aluminum is chosen as the mask material due to its small skin depth $(\delta \approx 14 \mathrm{~nm})$ and large surface plasmon propagation length $(\approx 3 \mu \mathrm{m}){ }^{[33]}$

The physical mechanisms of the extraordinary optical transmission by subwavelength holes have been extensively studied before. ${ }^{[34-38]}$ To tailor the transmission bands and polarization contrasts of the PMMs to match the absorption bands of photoalignment materials, we performed systematic simulations by using a commercial software package (CST MICROWAVE STUDIO). In the simulations, the hexahedral mesh scheme was used with mesh sizes $(\leq 10 \mathrm{~nm})$ much smaller than the surface plasmon wavelength; the dielectric permittivity of $\mathrm{Al}$ was obtained from the reference ${ }^{[39]}$ and the dielectric permittivity of $\mathrm{SiO}_{2}$ is fixed at 2.13 for all wavelengths. The thickness of the $\mathrm{SiO}_{2}$ substrates were set as infinity and the nanoaperture sizes and the Al film thicknesses were varied according to the designs. Polarized plane waves were illuminated from the glass substrate side. The electrical and magnetic fields in the plane located at $500 \mathrm{~nm}$ above the $\mathrm{Al}$ mask in the air side are calculated to represent the transmitted light intensity and polarization directions.

The simulation results suggest the following design preferences: (1) The triangular lattice is used over the square lattice because of its higher transmittance with the same aperture sizes and periods (Figure S2a,b, Supporting Information); (2) the mask film thickness is set at $150 \mathrm{~nm}$ as a trade-off between high transmittance and high polarization contrast (Figure S2c,d, Supporting Information); (3) Smaller array periodicity yields higher transmission but lower polarization contrast (Figure S3a,b, Supporting Information). (4) Nanoapertures around $100 \mathrm{~nm} \times 220 \mathrm{~nm}$ in size yield transmission bands and sufficient polarization contrasts for wavelengths between 400 and $600 \mathrm{~nm}$ (Figure S3c,d, Supporting Information). Based on the variations of the transmission peaks with the nanoaperture size and periodicity, the simulation results also suggest that the broadband optical transmissions result from two basic mechanisms: The surface plasmon resonance and the cavity resonance which are excited at two close but separated frequencies (Supporting Information). It can be verified that complex polarization patterns can be generated by arranging nanoapertures in arrays with spatially-varying orientation (Figure S4, Supporting Information).

Considering the reliability of the nanofabrication processes, we chose nanoapertures of $\approx 100 \mathrm{~nm} \times 220 \mathrm{~nm}$ in size as the optimal design. Numerical simulation results show that with $270 \mathrm{~nm}$ periodicity, these nanoaperture designs provide $\approx 40 \%$ optical transmission and a high polarization contrast ratio between 400 and $600 \mathrm{~nm}$ wavelengths (Figure 1a). To verify this design and the simulated optical properties, we fabricated parallel rectangular nanoaperture arrays of four different periodicities (see Figure $1 \mathrm{~b}$ as an example). The actual size of the fabricated nanoapertures is $\approx 100 \mathrm{~nm} \times 235 \mathrm{~nm}$. In agreements with the simulations (Figure 1a), the measured transmission spectra for polarization perpendicular to the long axis of these nanoapertures, i.e., transverse magnetic mode, exhibit broad peaks with the transmittance increasing with the reduction in the array periodicity (Figure 1c). The measured transmission for polarization parallel to the nanoapertures long axis, i.e., transverse electric mode is weak $(<5 \%)$, yielding a $>5 \mathrm{~dB}$ polarization contrast. To note, the measured transmission
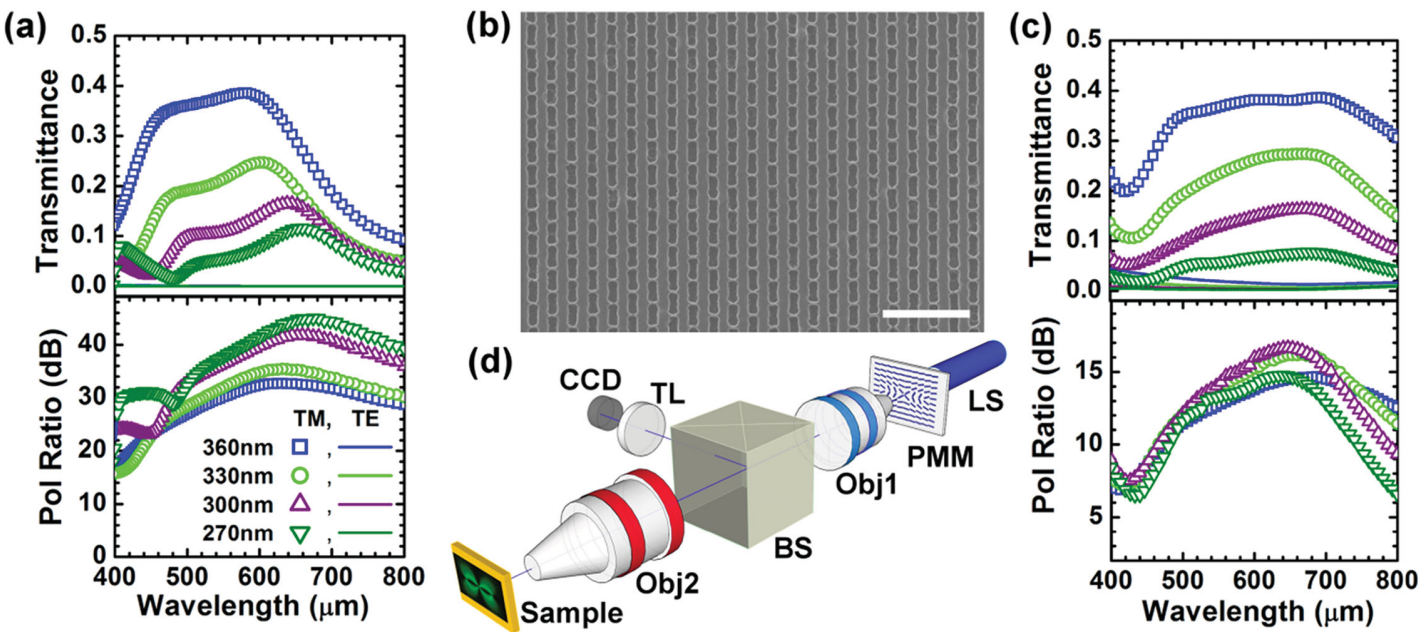

Figure 1. PMM design and projection photopatterning system. a) Simulated transmission (top) and polarization ratio (bottom) spectra for triangular lattices of parallel rectangular nanoapertures $(100 \mathrm{~nm} \times 220 \mathrm{~nm}$ in size) with four different periodicities. b) Exemplary SEM image of the parallel nanoaperture array with $270 \mathrm{~nm}$ periodicity. c) Measured transmission spectra and polarization ratio for parallel aperture arrays with different periodicities. d) Schematic projection photopatterning system. Obj1: imaging objective, Obj2: projection objective; LS: light source, TL: tube lens, BS: beam splitter. The scale bar in panel (b) is $1 \mu \mathrm{m}$. 
bandwidths are larger than those in numerical simulations obtained with normal illumination, because a condenser lens is used in the experiments to mimic mask illumination condition. In addition, it can be noted that the polarization contrasts measured in the experiments are lower than those obtained in the numerical simulations (Figure 1a). We attribute this discrepancy mainly to some imperfections in the nanofabricated PMMs, e.g., the $\mathrm{Al}$ inside some nanoapertures may not be completely etched.

As schematically shown in Figure 1d, we also developed a plasmonic projection photopatterning system for patterning the LC molecular director. A high power lamp of X-Cite series 120 is used as the light source for illuminating the plasmonic metamasks. The light transmitted through the metamasks is collected by the imaging objective (Obj1) and then coupled to the back aperture of the projection objective (Obj2). The projection objective focuses the mask patterns (along with their polarization fields) onto the LC cells or glass substrates. An optical microscope, which is made of the projection objective (Obj2), a tube lens, and a CCD camera, is used to inspect the LC cells and the mask patterns, to ensure (1) that the mask patterns are at focus in the LC cell plane and (2) that the mask patterns overlap with the marks on substrates. The magnification between the projected pattern and the original mask is approximately the ratio between the magnifications of the projection and imaging objectives.

As a proof-of-capability in patterning arbitrary director fields, we designed PMMs for clusters and arrays of topological defects in a nematic liquid crystal. The topological defects are used as examples because of two reasons. First, they are accompanied by strong gradients of the director fields and thus allow for testing the limits of the pattern designing and the projection photopatterning system. Second, the topological defects are central elements of numerous applications, such as directed colloidal assembly, ${ }^{[9-11]}$ programmable origami, ${ }^{[4-8]}$ and electrokinetic motion. ${ }^{[12-14]}$

2D director fields of a nematic LC satisfy the Laplace equation under single elastic constant approximation. ${ }^{[40]}$ For isolated single topological defects, the director orientation angle $\psi$ is a linear function of the azimuthal angle $\varphi:{ }^{[40]} \psi=k \varphi+\psi_{0}$, where $k$ represents the defect strength or topological charge. Based on this equation and the principle of superposition, we set the nanoaperture orientation at position $(x, y)$ in the metamasks for the defect clusters as $\psi=\sum_{i} k_{i} \tan ^{-1}\left(\frac{\gamma-\gamma_{i 0}}{x-x_{i 0}}\right)+\psi_{i 0}$, where $k_{i}, \psi_{i 0}, x_{i 0}$, and $\psi_{i 0}$ are respectively the defect charge, initial angle, $x$ and $y$ coordinates of the $i$-th defect core.

Figure 2a shows a representative SEM image of the fabricated PMM for a four defect flower where the defect at the center has $k_{1}=3 / 2, \psi_{10}=0$, while the defects at the periphery have $k_{i}=-1 / 2, \psi_{i 0}=0$ with $i=2,3,4$. The total defect charge is zero so the far-field director is uniform. Figure $2 \mathrm{~b}$ presents a transmission optical microscopic image of the PMM illuminated by nonpolarized white light. The optical transmission of the PMM exhibits spatial nonuniformity due to the complex interferences of surface plasmons and cavity modes and to some minor orientation dependence of the aperture size resulting from the fabrication processes. This nonuniformity does not affect photoalignment as overexposure by the high transmission regions does not change the predesigned alignment direction. Figure $2 \mathrm{c}$ presents the measured polarization patterns of the transmitted far-field light, where the local polarization direction is perpendicular to the long axis of the nanoapertures in the metamask. This is in good agreement with the design principle. The measured polarization contrasts range between 3 and 15 .
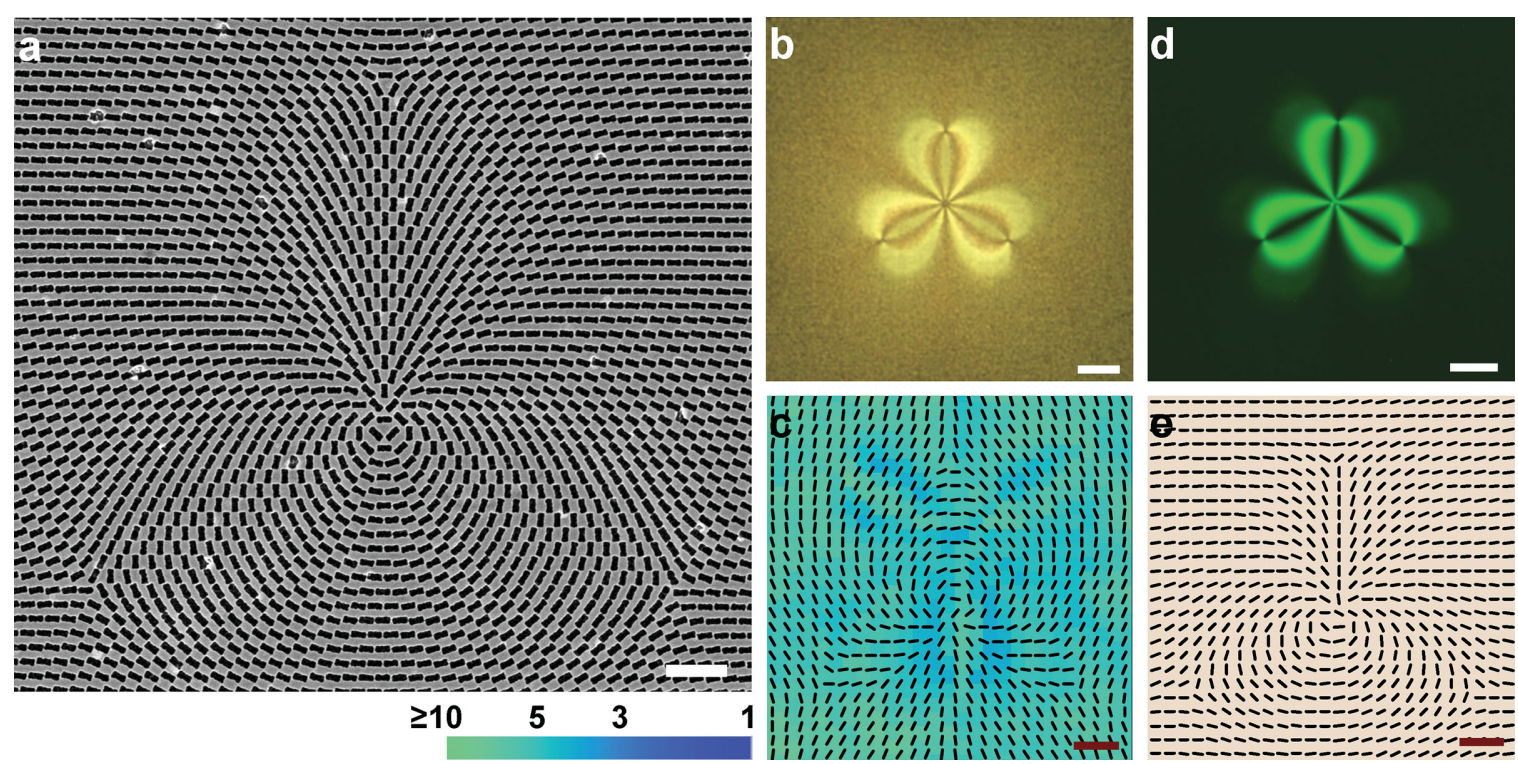

Figure 2. Plasmonic photopatterning of a four defect flower. a) SEM image of a PMM with a cluster of four topological defects. Here, $k_{1}=1.5, \psi_{10}=0, k_{i}=-0.5, \psi_{i 0}=0$ where $i=2-4$. For imaging clarity, the defect spacing is reduced in comparison to the real mask in (b). b) Transmission optical image of the PMM under illumination of nonpolarized white light. c) Measured polarization field for the PMM where the background color represents polarization contrast as indicated by the colored scale bar. d) Cross-polarized optical microscope of the four defects patterned with the mask. e) PolScope measured molecular orientation field. The scale bars in panels (a-e) are 1, 10, 5, 25, and $15 \mu \mathrm{m}$, respectively. The LC cell gap is $\approx 2 \mu \mathrm{m}$. 
For photopatterning, we project the transmitted light from the metamasks onto LC cells which are made of two parallel glass substrates with inner surfaces coated with photoalignment materials (Figure 1d). When the cell gap is within the field depth of the objectives, the top and bottom substrates can be exposed simultaneously. Depending on the numerical aperture (NA) of the objectives, the field depth, approximated as $\lambda / \mathrm{NA}^{2}$, can range from $2(0.5 \mathrm{NA})$ to $10 \mu \mathrm{m}(0.2 \mathrm{NA})$.

After photoexposure, the LC cells were filled with the $5 \mathrm{CB}$ nematic LC through capillary suction. Under a cross-polarized optical microscope, intriguing Schlieren textures can be observed in these photopatterned LC cells. For example, one $+3 / 2$ and three $-1 / 2$ defects exhibit the flower texture (Figure 2d) where the dark extinction brushes emanating from the defect cores indicate the regions where the LC director is either parallel or perpendicular to the polarizer. In these 2D patterns used in our work, the number $n_{\mathrm{b}}$ of dark brushes is related to the absolute charge of the defect $|k|=n_{\mathrm{b}} / 4$.
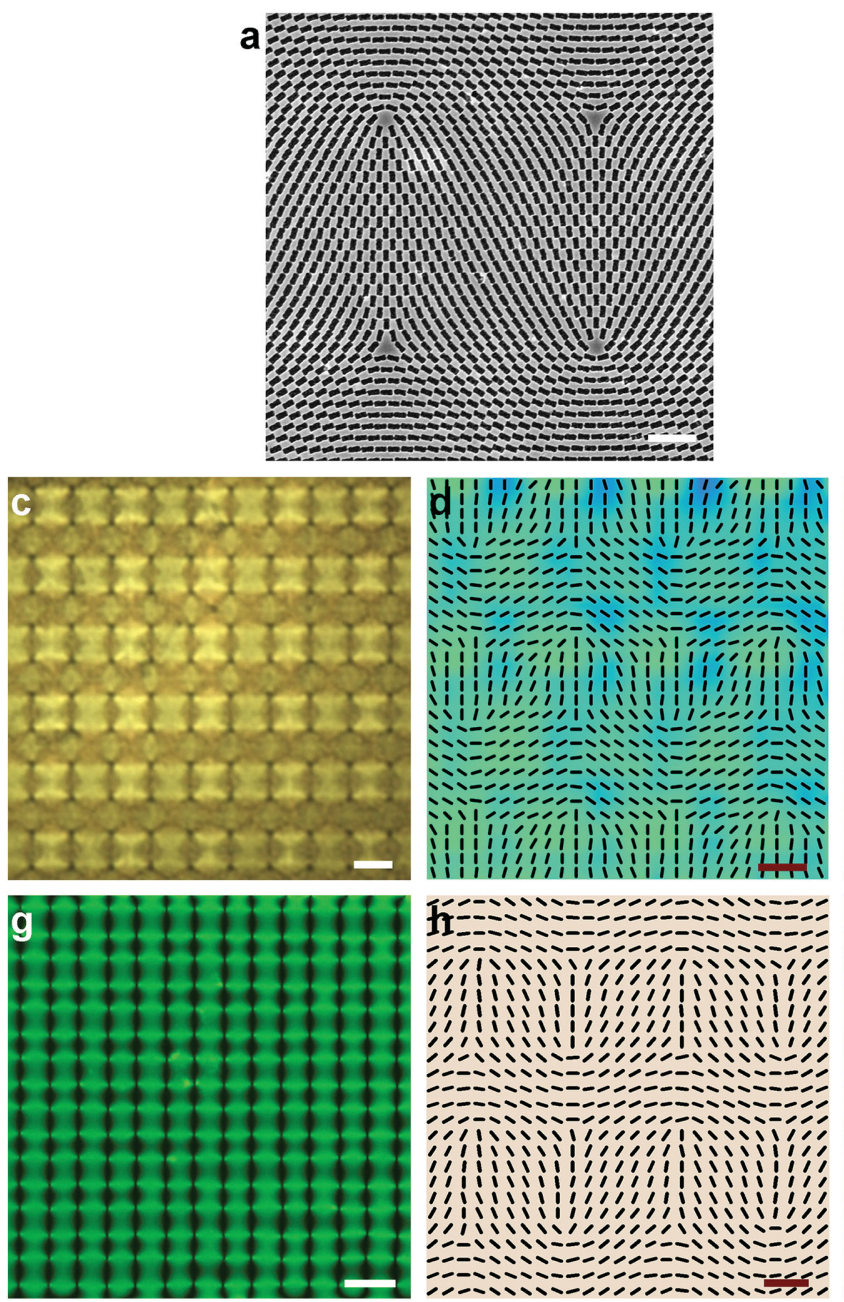

The molecular orientation in the patterned LC cells is mapped out by using the PolScope technique. During the PolScope microscopy, the samples are illuminated with a monochromatic light $(\lambda=546 \mathrm{~nm})$; by varying polarization direction of the illuminating light, the optical retardance $\Gamma(x, y)$ and the slow axis orientation of the birefringent $\mathrm{LC}$ can be mapped out. ${ }^{[41]}$ For $5 \mathrm{CB}$, the slow axis is just the long axis of rodshaped 5CB molecules. The PolScope results show that the patterned molecular director follows closely the nanoaperture orientations in the PMMs (Figure 2e; Figures S5-S7, Supporting Information). This verifies that the PMMs have an extremely simple design rule: Use the target molecular director fields as the design orientations of these apertures in the metamasks.

The metamasks for periodic arrays of topological defects are designed by tiling square patches of individual defects. As exemplified in Figure 3a,b, each unit cell is composed of two positive defects and two negative defects of the same
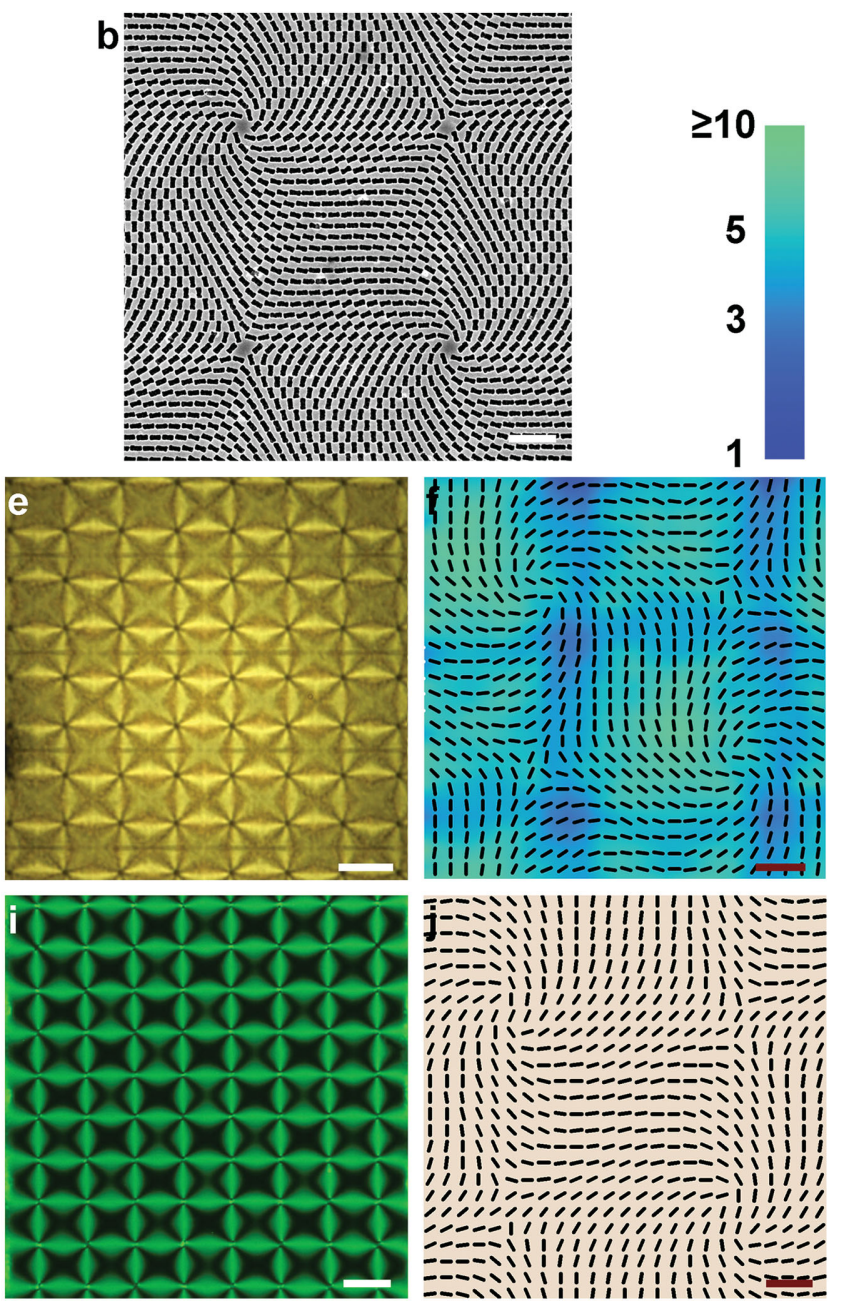

Figure 3. Plasmonic photopatterning of defect arrays. a,b) SEM images of the illustrative PMMs for $(1 / 2,-1 / 2)$ and $(+1,-1)$ topological defect arrays. The defect spacing is reduced from the real masks shown in panels (c) and (e) for imaging purpose. c,e) Transmission optical images of the PMMs under illumination of nonpolarized white light. d,f) Measured polarization fields for the PMMs c,e) where the background color represents polarization contrast as indicated by the colored scale bar. g,i) Cross-polarized optical microscopic images of liquid crystal textures patterned with the metamasks in panels (c) and (e). h,j) The LC director fields in panels (g) and (i) measured by a PolScope. The scale bars are $1 \mu \mathrm{m}$ in panels (a) and (b), $2.5 \mu \mathrm{m}$ in panels (d), (f), (h), and (j), $5 \mu \mathrm{m}$ in panel (c), $10 \mu \mathrm{m}$ in panel (e), (g), and $25 \mu \mathrm{m}$ in panel (i). The LC cell gaps are $\approx 2 \mu \mathrm{m}$. 
absolute charges. Optical characterization of these metamasks (Figure $3 c-f$ ) shows a good agreement with the design expectations: The polarization of the transmitted light is locally perpendicular to the nanoapertures' long axes. Photopatterning with such metamasks yields arrays of topological defects in LCs with absolute charges of $1 / 2,1,3 / 2$, and 2 (Figure 3g-j; Figure S8g-j, Supporting Information). The spatial maps of the LC director obtained through the PolScope show an excellent agreement with the orientation patterns of the nanoaperture long axes in the PMMs.

For the $(+1 / 2,-1 / 2)$ defect arrays, we used a $50 \times 0.55 \mathrm{NA}$ image objective and a $40 \times 0.6 \mathrm{NA}$ projection objective and achieved a $4.5 \mu \mathrm{m}$ spacing between the defects (Figure $3 \mathrm{~g}, \mathrm{~h}$ ). Given that the diameter of the diffraction Airy disk (1.22 $\lambda / \mathrm{NA})$ is $\approx 1 \mu \mathrm{m}$, this defect spacing is approaching the diffraction limit of the system. As a comparison, the highest resolution in maskless photopatterning techniques that allow for arbitrary director patterns is $2 \mu \mathrm{m}$ per pixel. ${ }^{[19]}$ It is important to point out that using interference patterns of coherent light beams can also yield very high spatial resolution in photoalignments, which provide a unique technique for certain specific set of periodic director patterns. ${ }^{[42,43]}$ In contrast, the plasmonic photopatterning technique offers the obvious advantage in aligning LCs in all possible periodic or nonperiodic director patterns with high resolution.

The projection system allows us to produce different director patterns at the top and bottom surfaces of the cells. Figure 4 shows two examples in which the top pattern is the same, composed of a square lattice of radial +1 and hyperbolic -1 point defects. The bottom director pattern is a square lattice of circular +1 and hyperbolic -1 point defects in the first case (Figure 4b) and is uniform in the second case (Figure 4c). In the first case, the LC microscopic texture shows a square lattice of disclinations (dark lines connecting the nearest point defect nodes in Figure $4 \mathrm{~b}$ ). This can be understood as splitting of the integer-strength defects in the bulk into pairs of semi-integer disclination lines. In the second case, there is only one subset of disclinations connecting neighboring defect sites along the $x$-axis direction, perpendicular to the $y$-axis alignment direction at the bottom plate (Figure $4 \mathrm{c}$ ). This freedom in aligning LC molecules into arbitrary director patterns at the top and bottom surfaces makes it possible to control the director fields of the
LCs in three dimensions, which will greatly enlarge the design spaces for applications such as LC elastomers for programmable origami, ${ }^{[7,8]}$ nonlinear electrokinetic flows in LCs, ${ }^{[14]}$ and LC-directed colloidal and molecular assembly. ${ }^{[44]}$

The plasmonic photopatterning method allows one to construct practically any director configuration, including defects with topological charges higher than these presented here. One should bear in mind, however, that because of the finite value of the photoinduced surface anchoring strength $W$, gradients of the director would be smoothed out on the characteristic length scale in the order of $K / W$, where $K$ is the relevant Frank constant or a combination of the Frank constants. The higher the $W$ is, the closer the photopatterned director is to the predesigned pattern. Determination of $W$ and its dependence on the photoalignment parameter is an important issue to explore in the future.

To conclude, we proposed and demonstrated a plasmonic projection photoalignment technique which enables unprecedented high resolution control of $2 \mathrm{D}$ and $3 \mathrm{D}$ molecular orientation with practically any designer complexity and topological strength. We would point out that the plasmonic photopatterning approach is advantageous over other existing techniques of liquid crystal alignment in several aspects. The mask-based single-exposure process ensures high throughput, repeatability, and low cost. Because of their similarity with the reticles in the projection photolithography, the PMMs are compatible with commercial stepper systems for wafer size exposure. Moreover, patterning area can be further enlarged by step-repeat exposures. This high-resolution, high-throughput photopatterning technique is expected to find unique applications in manufacturing various LC materials and devices whose functionalities rely on nonuniform molecular director fields.

\section{Experimental Section}

Fabrication of the PMMs: A $5 \mathrm{~nm}$ Ti adhesion layer and a $150 \mathrm{~nm} \mathrm{Al}$ film were sequentially deposited on fused quartz glass slides by e-beam evaporation, and then a $20 \mathrm{~nm} \mathrm{SiO}$ film was deposited by plasmaenhanced chemical vapor deposition. These glass substrates were then spin-coated with $100 \mathrm{~nm}$ films of poly-methyl-methacrylate (PMMA) as electron beam resist. After e-beam writing and resist development,
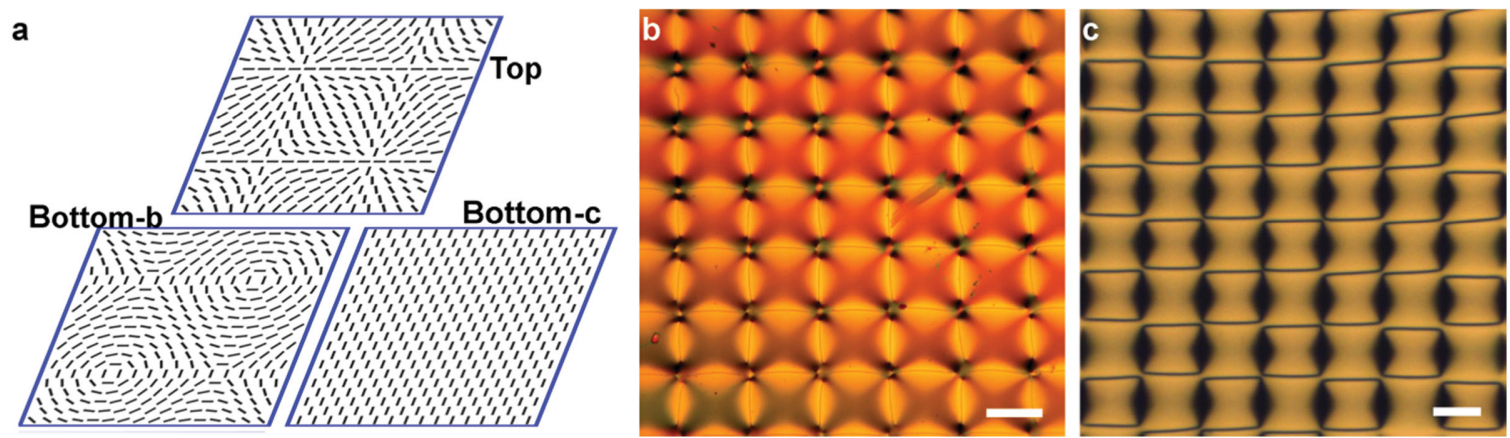

Figure 4. Plasmonic photopatterning of different top and bottom director fields. a) Schematic patterns: The top pattern is the same for (b) and (c), and the bottom pattern is arrays of +1 and -1 defects for (b) while uniform vertical alignment for (c). b) Cross-polarized optical microscope image of LC textures with a different defect array on the bottom. c) Cross-polarized optical microscopic image of LC textures with uniform director at the bottom. The scale bars in panels (b) and (c) are 200 and $20 \mu \mathrm{m}$, respectively. The LC cell gaps are $\approx 8 \mu \mathrm{m}$. 
the mask patterns in the PMMA films were transferred into the $\mathrm{SiO}_{2}$ layers through reactive ion etching with the following recipe: 10 SCCM $\mathrm{C}_{4} \mathrm{~F}_{8}, 174 \mathrm{SCCM} \mathrm{He}, 4 \mathrm{mTorr}$ pressure. In the last step, the patterned $\mathrm{SiO}_{2}$ layers were used as etching masks to transfer the $\mathrm{SiO}_{2}$ patterns into the aluminum films using the following recipe: $30 \mathrm{SCCM} \mathrm{BCl}_{3}$, $15 \mathrm{SCCM} \mathrm{Cl}_{2}$, and $4 \mathrm{SCCM} \mathrm{CH}_{4}$; and a pressure of 3 mTorr.

Liquid Crystal Materials and Cells: Glass slides were cleaned with detergent in an ultrasonic bath for half an hour and then exposed to UV ozone for $10 \mathrm{~min}$ to promote the hydrophilicity. The glass slides were then spin-coated with brilliant yellow (BY, $0.5 \mathrm{wt} \%$ in dimethylformamide) or PAAD (use as received from Beam Co.), and baked on a $90{ }^{\circ} \mathrm{C}$ hot plate for $20 \mathrm{~min}$. For the spin-coating, $500 \mathrm{rpm} \mathrm{s}^{-1}$ acceleration, $1500 \mathrm{rpm}$ spin speed, and $40 \mathrm{~s}$ of spin time were used, and the final thicknesses of the BY films were measured to be $\approx 8 \mathrm{~nm}$. Two glass substrates with the coated inner surfaces were assembled to form a liquid crystal cell. The cell thickness was controlled using fiber spacers, and ranged between 1.7 and $2.5 \mu \mathrm{m}$. For photopatterning different directors at two sides of the cells, first marks on the top and bottom substrates were fabricated and then coated them with photoalignment materials. The top and bottom substrates were patterned separately at positions predesigned with respect to the marks, and then assembled into LC cells with the top and bottom marks aligned. The cell thickness for this case was kept at $\approx 8 \mu \mathrm{m}$. The exposure time required for photopatterning is dependent on the light illumination conditions and especially the magnification of the patterning. In most experiments, the photoexposure time used is ranged between $2 \mathrm{~s}$ for the $1.25 \times$ magnification and $2 \mathrm{~min}$. for the 10x magnification. Here, the threshold exposure time needed for the $1.25 \times$ magnification should be much shorter than $2 \mathrm{~s}$.

\section{Supporting Information}

Supporting Information is available from the Wiley Online Library or from the author.

\section{Acknowledgements}

This work was supported by the National Science Foundation through CMMI-1436565 and DMR-1507637. O.Y. acknowledges support from Fulbright Foundation under the program G-1-00005. The author Q.-H.W. likes to acknowledge valuable discussions with Philip J. Bos, Wu Lu, Carl D. Modes, Charles Rosenblatt, Robin B. Selinger, Timothy J. White, and Hiroshi Yokoyama.

Received: December 3, 2015 Revised: December 19, 2015 Published online: January 22, 2016

[1] P. F. McManamon, P. J. Bos, M. J. Escuti, J. Heikenfeld, S. Serati, X. Huikai, E. A. Watson, Proc. IEEE 2009, 97, 1078.

[2] L. Marrucci, C. Manzo, D. Paparo, Phys. Rev. Lett. 2006, 96,163905

[3] S. R. Nersisyan, N. V Tabiryan, D. M. Steeves, B. R. Kimball, Opt. Photonics News 2010, 21, 40.

[4] L. T. De Haan, C. Sánchez-Somolinos, C. M. W. Bastiaansen, A. P. H. J. Schenning, D. J. Broer, Angew. Chem. Int. Ed. 2012, 51, 12469.

[5] M. E. McConney, A. Martinez, V. P. Tondiglia, K. M. Lee, D. Langley, I. I. Smalyukh, T. J. White, Adv. Mater. 2013, 25, 5880.

[6] L. T. de Haan, V. Gimenez-Pinto, A. Konya, T.-S. Nguyen, J. M. N. Verjans, C. Sánchez-Somolinos, J. V Selinger, R. L. B. Selinger, D. J. Broer, A. P. H. J. Schenning, Adv. Funct. Mater. 2014, 24, 1251.

[7] T. H. Ware, M. E. McConney, J. J. Wie, V. P. Tondiglia, T. J. White, Science 2015, 347, 982.
[8] T. J. White, D. J. Broer, Nat. Mater. 2015, 14, 1087.

[9] D. Voloschenko, O. P. Pishnyak, S. V. Shiyanovskii, O. D. Lavrentovich, Phys. Rev. E 2002, 65, 60701.

[10] I. Muševič, M. Škarabot, U. Tkalec, M. Ravnik, S. Žumer, Science 2006, 313, 954.

[11] M. Cavallaro, M. A. Gharbi, D. A. Beller, S. Čopar, Z. Shi, T. Baumgart, S. Yang, R. D. Kamien, K. J. Stebe, Proc. Natl. Acad. Sci. USA 2013, 110, 18804.

[12] O. D. Lavrentovich, I. Lazo, O. P. Pishnyak, Nature 2010, 467, 947.

[13] I. Lazo, C. Peng, J. Xiang, S. V. Shiyanovskii, O. D. Lavrentovich, Nat. Commun. 2014, 5, 5033.

[14] C. Peng, Y. Guo, C. Conklin, J. Viñals, S. V. Shiyanovskii, Q.-H. Wei, O. D. Lavrentovich, Phys. Rev. E 2015, 92, 52502.

[15] K. Ichimura, Chem. Rev. 2000, 100, 1847.

[16] V. G. Chigrinov, V. M. Kozenkov, H.-S. Kwok, Photoalignment of Liquid Crystalline Materials: Physics and Applications, John Wiley \& Sons Ltd., West Sussex, England 2008.

[17] O. Yaroshchuk, Y. Reznikov, J. Mater. Chem. 2012, 22, 286.

[18] B. S. Murray, R. A. Pelcovits, C. Rosenblatt, Phys. Rev. E 2014, 90, 52501.

[19] C. Culbreath, N. Glazar, H. Yokoyama, Rev. Sci. Instrum. 2011, 82, 126107.

[20] H. Wu, W. Hu, H. Hu, X. Lin, G. Zhu, J.-W. Choi, V. Chigrinov, Y. Lu, Opt. Express 2012, 20, 16684.

[21] A. V. Kildishev, A. Boltasseva, V. M. Shalaev, Science 2013, 339, 6125.

[22] N. Yu, F. Capasso, Nat. Mater. 2014, 13, 139.

[23] N. Yu, P. Genevet, M. A. Kats, F. Aieta, J.-P. Tetienne, F. Capasso, Z. Gaburro, Science 2011, 334, 333.

[24] X. Ni, N. K. Emani, A. V. Kildishev, A. Boltasseva, V. M. Shalaev, Science 2012, 335, 427.

[25] P. Genevet, N. Yu, F. Aieta, J. Lin, M. A. Kats, R. Blanchard, M. O. Scully, Z. Gaburro, F. Capasso, Appl. Phys. Lett. 2012, 100, 13101.

[26] Y. Yang, W. Wang, P. Moitra, I. I. Kravchenko, D. P. Briggs, J. Valentine, Nano Lett. 2014, 14, 1394.

[27] A. Arbabi, Y. Horie, M. Bagheri, A. Faraon, Nat. Nanotechnol. 2015, 10,937.

[28] D. Lin, P. Fan, E. Hasman, M. L. Brongersma, Science 2014, 345, 298.

[29] G. Zheng, H. Mühlenbernd, M. Kenney, G. Li, T. Zentgraf, S. Zhang, Nat. Nanotechnol. 2015, 10, 308.

[30] F. Aieta, P. Genevet, M. A. Kats, N. Yu, R. Blanchard, Z. Gaburro, F. Capasso, Nano Lett. 2012, 12, 4932

[31] Z.-W. Liu, Q.-H. Wei, X. Zhang, Nano Lett. 2005, 5, 957.

[32] W. Srituravanich, L. Pan, Y. Wang, C. Sun, D. B. Bogy, X. Zhang, Nat. Nanotechnol. 2008, 3, 733.

[33] S. G. Rodrigo, F. J. García-Vidal, L. Martín-Moreno, Phys. Rev. B 2008, 77, 75401

[34] T. W. Ebbesen, H. J. Lezec, H. F. Ghaemi, T. Thio, P. A. Wolff, Nature 1998, 391, 667.

[35] F. J. Garcia-Vidal, L. Martin-Moreno, T. W. Ebbesen, L. Kuipers, Rev. Mod. Phys. 2010, 82, 729.

[36] K. L. van der Molen, K. J. Klein Koerkamp, S. Enoch, F. B. Segerink, N. F. van Hulst, L. Kuipers, Phys. Rev. B 2005, 72,45421.

[37] Z. Ruan, M. Qiu, Phys. Rev. Lett. 2006, 96, 233901.

[38] A. Mary, S. G. Rodrigo, L. Martín-Moreno, F. J. García-Vidal, Phys. Rev. B 2007, 76, 195414.

[39] E. D. Palik, E. D. Palik, Handbook of Optical Constants of Solids, Academic Press, San Diego 1998.

[40] J. Nehring, A. Saupe, J. Chem. Soc., Faraday Trans. 2 1972, 68, 1.

[41] M. Shribak, R. Oldenbourg, Appl. Opt. 2003, 42, 3009.

[42] E. A. Shteyner, A. K. Srivastava, V. G. Chigrinov, H.-S. Kwok, A. D. Afanasyev, Soft Matter 2013, 9, 5160.

[43] T. Du, F. Fan, A. M. W. Tam, J. Sun, V. G. Chigrinov, H. S. Kwok, Adv. Mater. 2015, 27, 7191.

[44] X. Wang, D. S. Miller, E. Bukusoglu, J. J. De Pablo, N. L. Abbott, Nat. Mater. 2015, 15, 106. 